Original Research Paper

\title{
A Comparative Study on Barrier Option Pricing using Antithetic and Quasi Monte-Carlo Simulations
}

\author{
Nneka Umeorah and Phillip Mashele \\ Unit for Business Mathematics and Informatics, \\ North-West University, Potchefstroom, South Africa
}

Article history

Received: 26-01-2018

Revised: 22-02-2018

Accepted: 07-05-2018

Corresponding Author:

Nneka Umeorah

Unit for Business

Mathematics and Informatics,

North-West University,

Potchefstroom, South Africa

Email:

umeorahnnekaozioma@gmai

1.com

\begin{abstract}
Monte-Carlo simulations have been utilized greatly in the pricing of derivative securities. Over the years, several variance reduction techniques have been developed to curb the instability, as well as, increase the simulation efficiencies of the Monte-Carlo methods. Our approach in this research work will consider the use of antithetic variate techniques to estimate the fair prices of barrier options. Next, we use the quasi-Monte Carlo method, together with Sobol sequence to estimate the values of the same option. An extended version of the Black-Scholes model will serve as basis for the exact prices of these exotic options. The resulting simulated prices will be compared to the exact prices. The research concludes by showing some results which proves that when random numbers are generated via low discrepancy sequences in contrast to the normal pseudo-random numbers, a more efficient simulation method is ensued. This is further applicable in pricing complex derivatives without closed formsolutions.
\end{abstract}

Keywords: Black-Scholes Model, Barrier Options, Monte-Carlo, Antithetic Monte-Carlo, Quasi Monte-Carlo, Sobol Sequence, 2010 Mathematics Subject Classification: 91G60, 91G20, 91G30

\section{Introduction}

The path-dependent nature of barrier options classified them as exotic options and this becomes difficult during delta-hedging. However, notwithstanding their complicated nature of pricing, investors and corporate firms had used barrier options in portfolio insurance framework and bond trading, thereby improving efficiency and eliminating possible transaction costs (Rich, 1994, p. 295). With regards to bond trading, Cox and Rubinstein (1985, p. 408-411) explained that bonds which are fixed with short Downand-Out (DO) barrier call options can be used to minimize the costs related to writing and imposing expensive bond agreements. Barrier options, as exotic options are traded both on the standardized exchange and in the over-the-counter market. As important financial instruments, Luenberger and Luenberger (1999) explained that barrier options trading account for " $50 \%$ of the volume of all exotic options and $10 \%$ volume of all traded securities". Barrier options are options whose payoffs depend on the barrier levels that the underlying prices attain to during the life of the contract. These options can be classified into up(down) options if the barrier level is positioned above(below) the underlying price respectively. Research works have been done in the class of barrier options, but relatively no such work has been carried out on the comparative study focusing on the use of antithetic and quasi Monte-Carlo simulation method to price zero-rebate barrier call option with European knock-out features. Hence, this research seeks to address the gap.

One of the earliest research on barrier option pricing can be found in Snyder (1969). He described the DO options as limited risk special options, in which the holder agrees (with a favorable price in return) to limit the risk experienced by the writer by making the option void supposing "the price of the stock declines during the life of the contract to a specific point below the striking price called the expiration price". Furthermore, Merton (1973) priced the DO barrier options using the Partial Differential Equations (PDE) approach. Pricing discrete barrier options are also found in works of literature (Kou, 2003; Broadie et al., 1997). Zvan et al. (2000) presented an implicit method for solving PDE models in relation to barrier options. An explicit formula 
for obtaining the knock-out discount for barrier options can be obtained in Musiela and Rutkowski (2006). On the conditional Monte-Carlo simulations, Brandimarte (2013) analyzed the problem and applied it to DO put options. Different variance reduction techniques applied in the Monte-Carlo methods on option pricing were reviewed by Bolia and Juneja (2005). They employed different recent Monte-Carlo techniques like the regression-based, random tree and the stochastic mesh methods specifically on Bermudan and American options. Rich (1994) presented a mathematical foundation, mostly the key density function approach which is in connection to the solution of the FokkerPlank PDE, to value the barrier options. He derived closed form solutions of the European barrier options possessing a rebate and a fixed barrier level. A rebate is positive discount paid to the option holder incase of an a knock-out or knock-in and its presence increases the value of the barrier option, even though it has no effect on its payoff. Furthermore, Alzubaidi (2016) employed the concept of antithetic variate, together with the Brownian bridge to improve the efficiency of the MonteCarlo methods in the valuation of rebate barrier options.

\section{Practical Applicability of the Monte-Carlo Methods}

The MCS methods are techniques for obtaining numerical solutions to certain problems using some computational algorithm that focuses on series of iterative random sampling. Kroese et al. (2014) explained that Monte-Carlo methods are applied mainly in three welldefined problem classes, which are sampling, estimation and optimization. Consider the evolution of stock prices in the financial market, for example, they follow a randomized system. Thus, the Monte-Carlo methods are introduced artificially to solve deterministic functions by which corresponding samples are drawn from a given probability distribution. Depending on the complexity of the dynamical system models, large number of samples are required in the simulation using the Monte-Carlo methods. These samples duly depend on large computation time. The concept of variance reduction method results in greater efficiency, with respect to the convergence process, as well as to an acceleration of the simulation techniques (Roboam, 2012). Variance reduction techniques include but not limited to antithetic variates, correlated sampling, importance sampling, control variates and stratified sampling.

Application of Antithetic Monte-Carlo Simulation (AMCS) methods can be seen in the fields of science, finance and engineering. Carmona et al. (2012) explained that Monte-Carlo methods are applicable to credit risk analysis, dynamic parameter derivatives and option pricing in the field of finance. It is also used in the estimation of fixed income instruments and interest rate derivatives which are defined in diverse term structure models. These are mostly used in situations where the closed form solutions of these securities are non-existent. Risk and uncertainties of certain projects can be managed by the aid of the Monte-Carlo methods and this had greatly improved its use in risk management. Glasserman (2004) asserted that the AMCS had played a significant role in the measurement of risks that are associated with a given portfolio of assets, as well as in the measurement of profit and loss distribution of a portfolio.

Certain complex problems in finance, statistics, technology, economics and probability theorems which cannot be resolved by the normal mathematical methods can be resolved using the quasi Monte Carlo simulation methods (QMCS). The QMCS combines methodologies and results from algebra, combinatorics and number theory to quantitatively generate number points which can be used for simulation purposes. Furthermore, the multidimensional numerical integration which are evident in financial problems are handled properly when the QMCS method is used. Fang (2002) discussed some of the applications of the QMCS methods to statistics and they were linked to the resolution of problems in statistical inference, geometric probability, experimental designs, as well as, Bayelsian statistics. Caisch (1998) analyzed the applications of QMCS to the field of scientific computing and it was observed that the rate of convergence for the QMCS is asymptotically faster than the normal MCS. Au Yeung (2010) focused on Asian option pricing using the quasi Monte-Carlo methods, in connection with MATLAB.

The significance of this research is to conduct a comparative study on zero rebate knock-out barrier option pricing using the MCS, AMCS and QMCS methods. We further seek generalize which Monte-Carlo methods show higher efficiency in valuing the pathdependent barrier options so as to avoid underpricing. This research, however, will focus on how financial risk can be hedged using exotic option pricing and these prices will be obtained using the techniques of the MCS, AMCS and QMCS methods. It will further explain how the method can be incorporated in evaluating financial derivatives, like the path-dependent barrier options. The organization of this research is as follows: Section 1 introduced the study, with brief literature studies on barrier option valuation using numerical approximations. It further discussed the practical applicability of the Monte-Carlo methods. Section 2 considers the theoretical valuation for both the DO and the up-and-out (UO) non-dividend barrier call options. The closed form solutions of these existing formulas are properly referenced. Section 3 discusses the numerical approaches to the knock-out barrier option valuation, which in this context refers to the ordinary MCS, AMCS and QMCS methods. Section 4 provides the numerical results and the corresponding comparative statics, whereas Section 5 concludes the study. 


\section{Valuation of Zero-Rebate Knock-Out Barrier Options}

Generally, a barrier option with a knock-out feature refers to an option which ceases to exist once the barrier level is being hit by the price of an underlying asset. Thus, the option holder receives a rebate (positive discount) immediately or at maturity if the barrier is triggered within the contract's life, but receives the value of European option if the underlying never touches the barrier.

\section{Definition 2.1. (Zero-Rebate Knock-Out Barrier Options) (Zhang, 1998)}

A zero-rebate knock-out barrier option pays nothing if the barrier is reached but pays the value of the European option if otherwise. It is also referred to as an option whose payoff at time $T$ is defined by:

$$
f(S(T))\left(1-\mathbb{I}_{\tau<T}\right) .
$$

Here, $\tau$ which is a function of the underlying $S$ and the barrier level $B$, denotes the stopping time for the barrier; $f$ is the payoff function, depending on the type of option and finally, $\mathbb{I}_{\lambda}$ is an indicator function which pays 1 when the event $\lambda$ (breaching of the barrier) occurs or 0 otherwise.

Terminal and Boundary Conditions for ZeroRebate Knock-Out Barrier Options

Let $f(T, S)$ be the payoff function of the DO barrier call option and $V(t, S)$ be the value of the non-dividend DO barrier call option, then the option value $V(t, S)$ satisfies the Black-Scholes PDE given below:

$$
\begin{aligned}
& \frac{\partial V(t, S)}{\partial t}+r S \frac{\partial V(t, S)}{\partial S} \\
& +\frac{S^{2} \sigma^{2}}{2} \frac{\partial^{2} V(t, S)}{\partial S^{2}}-r V(t, S)=0,
\end{aligned}
$$

with the terminal and boundary conditions given as:

$$
\begin{aligned}
& V(T, S)=f(T, S)=\max \{S(T)-K, 0\} \\
& V(t, B)=0 \\
& V(t, \infty) \sim S-K e^{-r(T-t)} .
\end{aligned}
$$

Here $T$ refers to the maturity time; $t$, the current time; $r$, the risk-free interest rate; $\sigma$, the volatility rate; $S$, the current price of the underlying asset; $S(T)$, the price of the underlying asset at expiry; $K$, the strike price and $B$, the barrier level. Equation 2.2 gives the payoff or the terminal value of the option, the condition in Equation 2.3 occurs when the minimum value of the underlying asset price coincides with the barrier level and Equation 2.4 is when the underlying price attains its maximum.

The UO call option knocks-out once the maximum value of the underlying asset price coincides with the barrier level within the contract's lifespan. Let $V^{*}(t, S)$ denote its value at time $t$. Then, the option can be priced using the same PDE above but the terminal and boundary conditions become:

$$
\begin{aligned}
& V^{*}(T, S)=\max \{S(T)-K, 0\} \\
& V^{*}(t, B)=0 \\
& V^{*}(t, 0) \sim 0 .
\end{aligned}
$$

The PDE in Equation 2.1 can be solved using the hedging analysis of Black and Scholes. The equation is converted to heat equation, whereby the valuations of vanilla options can be related to the flow of heat in an infinite bar and this is solved by the method of images with respect to the standard European options (Buchen, 2012; Wilmott et al., 1995).

\section{Valuation of Zero-Rebate Down-and-Out Call Option}

The domain considered when valuating the DO call options is given by:

$$
D=\{(t, S): B<S<\infty, t \in[0, T]\}
$$

\section{Corollary 2.2. (Buchen, 2012)}

Let $v(t, S)$ be the value of a European call option, then the image of the function $v(t, S)$ with respect to assumed barrier level $S=B$ and the Black-Scholes differential operator is given by:

$$
v^{*}(t, S)=\left(\frac{B}{S}\right)^{\alpha} v\left(t, \frac{B^{2}}{S}\right)
$$

where, $\alpha=\frac{2 r}{\sigma^{2}}-1$.

\section{Theorem 2.3. (Buchen, 2012)}

Let $v(t, S)$ be the solution of the terminal value problem de-scribed as:

$$
\begin{aligned}
& \mathcal{L} v(t, S)=0 \\
& v(T, S)=f(T, S) \mathbb{I}_{\{S>B\}},
\end{aligned}
$$

in the domain $\{S>0, t<T\}$, where $\mathcal{L} v(t, S)$ is the BlackScholes differential operator found in Equation 2.1. $f(T, S)$ 
is the payoff function at Equation 2.2 and $\mathbb{I}_{\{S>B\}}$ is an indicator function which outputs 1 when $S>B$ and 0 , otherwise. Then, the DO call value:

$$
V(t, S)=v(t, S)-v^{*}(t, S)
$$

solves the terminal boundary value problem for the DO barrier option in the domain $\{S>B, t<T\}$.

According to Hull (2006), the extended BlackScholes equation which gives the exact or the theoretical value for DO at time $t=0$ when $B>K$ is given by (For the put option values, see Hull (2006)):

$$
\begin{aligned}
& V(t, S)=S(0) N\left(x_{1}\right)-K e^{-r T} N\left(x_{1}-\sigma \sqrt{T}\right) \\
& -S(0)\left(\frac{B}{S(0)}\right)^{2 \lambda} N\left(y_{1}\right) \\
& +K e^{-r T}\left(\frac{B}{S(0)}\right)^{2 \lambda-2} N\left(y_{1}-\sigma \sqrt{T}\right),
\end{aligned}
$$

where:

$$
\begin{aligned}
x_{1}= & \frac{\ln \left(\frac{S(0)}{B}\right)}{\sigma \sqrt{T}}+\lambda \sigma \sqrt{T}, \\
y_{1}= & \frac{\ln \left(\frac{B}{S(0)}\right)}{\sigma \sqrt{T}}+\lambda \sigma \sqrt{T}, \lambda=\frac{r+\frac{\sigma^{2}}{2}}{\sigma^{2}}
\end{aligned}
$$

For $B \leq K$, the price becomes:

$$
\begin{aligned}
& V(t, S)=S(0) N\left(d_{1}\right)-K e^{-r T} N\left(d_{2}\right) \\
& -S(0)\left(\frac{B}{S(0)}\right)^{2 \lambda} N(y) \\
& +K e^{-r T}\left(\frac{B}{S(0)}\right)^{2 \lambda-2} N(y-\sigma \sqrt{T}),
\end{aligned}
$$

Where:

$$
\begin{aligned}
& d_{1}=\frac{\ln \left(\frac{S(0)}{K}\right)}{\sigma \sqrt{T}}+\lambda \sigma \sqrt{T}, \\
& d_{2}=d_{1}-\sigma \sqrt{T} \\
& y=\frac{\ln \left(\frac{B^{2}}{S(0) K}\right)}{\sigma \sqrt{T}}+\lambda \sigma \sqrt{T} .
\end{aligned}
$$

\section{Valuation of Zero-Rebate UO Call Option}

The domain under consideration for this class of knock-out options is:

$$
D=\{(t, S): 0 \leq S<B, t \in[0, T]\}
$$

According to Hull (2006), the extended BlackScholes equation which gives the exact value for $\mathbf{U O}$ at time $t=0$ when $B>K$ is given by (For the put option values, see Hull (2006)):

$$
\begin{aligned}
& V^{*}(t, S)=S(0) N\left(d_{1}\right)-K e^{-r T} N\left(d_{2}\right) \\
& -\left[\begin{array}{l}
S(0) N\left(x_{1}\right)-K e^{-r T} N\left(x_{1}-\sigma \sqrt{T}\right)-S(0)\left(\frac{B}{S(0)}\right)^{2 \lambda} \\
{\left[N(-y)-N\left(-y_{1}\right)\right]+K e^{-r T}\left(\frac{B}{S(0)}\right)^{2 \lambda-2}} \\
{\left[N(-y+\sigma \sqrt{T})-N\left(-y_{1}+\sigma \sqrt{T}\right)\right]}
\end{array}\right]
\end{aligned}
$$

Lastly, when $B \leq K$, we have that:

$V^{*}(t, S)=0$.

Equation 2.11 follows from the fact that the strike price is normally positioned below the barrier. But supposing that it is fixed above the barrier level, the option becomes worthless by the time it hits the barrier. Thus, the chances of the option expiring in the money become negligible.

We note that in Equations 2.9 and 2.10, the first two terms denote the Black-Scholes formula for the European call options. Likewise the first two terms in Equation 2.8, but with $B=K$. Furthermore, the remaining terms denote that extra condition with regards to the barrier feature, that is, the risk of the option being knocked-out with the sudden loss of the premium.

\section{Numerical Approximations}

This section gives an overview of the simulation techniques employed by the standard MCS, AMCS and the QMCS methods to barrier option pricing.

\section{Antithetic Monte-Carlo Simulation}

Generally, the MCS is a statistical estimation method which is based on the generation of random numbers. Boyle et al. (1997) first used the MCS to price the European options under the Black-Scholes assumptions (Black and Scholes, 1973). The MCS of option pricing under the Black-Scholes framework involves the generation of $N$ sample independent asset price movements and then the estimation of their payoffs. These payoffs are averaged and then discounted at a riskfree interest rate. One of the major setbacks of using the MCS is seen in its slow rate of convergence at which the 
estimated values tend to the true solution. This can be explained by the large variances obtained after the simulation. It is equally observed that the confidence interval obtained using the MCS is greatly influenced by the ratio of the standard deviation to the square root of the number of simulations. To further reduce the width of the interval, the deviation or the variance has to be reduced which in turn gives a better estimate.

The AMCS was introduced in this study to improve the flaws of the MCS. According to Glasserman (2004), the AMCS focuses on the symmetric properties of normal distribution to reduce the variance of the results been simulated and this is achieved by introducing negatively correlated random variables. Using a set of random numbers which consists of a positive and a negative number, say $x_{1}$ and $x_{2}$ respectively, the option value can be estimated. Let $X$ be a random variable whose estimate is unknown. Also, let $x_{1}$ and $x_{2}$ be two negatively correlated variables with the same mean $\mu$ and variance $\sigma^{2}$. Define:

$$
X=\frac{1}{2}\left(x_{1}+x_{2}\right)
$$

then the mean and the variance are given respectively as:

$$
\begin{aligned}
& \mathbb{E}[X]=\mathbb{E}\left[\frac{1}{2}\left(x_{1}+x_{2}\right)\right]=\frac{1}{2}\left(\mathbb{E}\left[x_{1}\right]+\mathbb{E}\left[x_{2}\right]\right)=\mu . \\
& \operatorname{var}[X]=\operatorname{var}\left[\frac{1}{2}\left(x_{1}+x_{2}\right)\right] \\
& =\frac{1}{4}\left(\operatorname{var}\left[x_{1}\right]+\operatorname{var}\left[x_{2}\right]+2 \operatorname{cov}\left[x_{1}, x_{2}\right]\right) \\
& =\frac{1}{2}\left(\operatorname{var}\left[x_{1}\right]+\operatorname{cov}\left[x_{1}, x_{2}\right]\right) .
\end{aligned}
$$

Thus, we have that:

$$
\operatorname{var}[X]\left\{\begin{array}{l}
=\frac{\sigma^{2}}{2} \text { if } x_{1} \text { and } x_{2} \text { are identicallyindependent }(\operatorname{cov}=0) \\
<\frac{\sigma^{2}}{2} \text { if } \operatorname{cov}\left[x_{1}, x_{2}<0\right]
\end{array}\right.
$$

It is essential that if the $\operatorname{cov}\left[x_{1}, x_{2}\right]<0$ or $\operatorname{cov}\left[x_{1}, x_{2}\right]=0$, then the variance is reduced. For the outputs of the antithetic variates to be negatively correlated, it is essential that the inputs are negatively correlated. The mapping between them should be monotone. Non-monotonic functions result in a non-negative correlation and this could increase the variance, instead of reducing as expected.

\section{Definition 3.1. (Monotone Functions)}

Let $A$ be a subset of $\mathbb{R}$ and define a function $g: A \rightarrow$ $\mathbb{R}$ and $x, y \in A$. Then $g$ is monotonic if $x<y$ implies $g(x) \leq g(y)$ or if $x<y$ implies $g(x) \geq g(y)$. The following corollaries help in the generation of negatively correlated random variables which are uniformly and normally distributed.

\section{Corollary 3.2. (Chan and Wong, 2015)}

If $g\left(X_{1}, X_{2}, \cdots, X_{n}\right)$ is a monotone function of each of its argument. Then the following holds for a set $\eta_{1}, \cdots, \eta_{m}$ of independent and identically distributed uniformly random numbers on $(0,1)$ :

$$
\operatorname{cov}\left[g\left(\eta_{1}, \cdots, \eta_{m}\right), g\left(1-\eta_{1}, 1-\eta_{2}, \cdots, 1-\eta_{m}\right)\right] \leq 0 .
$$

Let $\eta_{1}, \cdots, \eta_{m}$ and $\beta_{1}=1-\eta_{1}, \cdots, \beta_{m}=1-\eta_{m}$ be uniformly random numbers with the properties that the pair $\left(\eta_{1}, \beta_{1}\right)$ are negatively correlated. Define a function $g$ to be monotone. If $X_{1}=g\left(\eta_{1}, \cdots, \eta_{m}\right)$, then $X_{2}=g\left(\beta_{1}, \cdots, \beta_{m}\right)$ must be defined in the same distribution as $X_{1}$. Thus, from Corollary 3.2, we have that $\operatorname{cov}\left(X_{1}, X_{2}\right) \leq 0$. Hence, $X_{1}$ and $X_{2}$ are negatively correlated.

\section{Corollary 3.3. (Chan and Wong, 2015)}

If $g\left(X_{1}, X_{2}, \cdots, X_{n}\right)$ is a monotone function of each of its argument. Then the following holds for a set $\eta_{1}, \cdots, \eta_{m}$ of independent and identically distributed normal random numbers on $(0,1)$ :

$$
\operatorname{cov}\left[g\left(\eta_{1}, \cdots, \eta_{m}\right), g\left(-\eta_{1},-\eta_{2}, \cdots,-\eta_{m}\right)\right] \leq 0 .
$$

Let $X_{i} \sim N\left(\mu, \sigma^{2}\right)$ and $Y_{i}=2 \mu-X_{i}$. Then from the linearity condition of the normal random variable, we see that $Y_{i} \sim N\left(\mu, \sigma^{2}\right)$. Thus, $X_{i}$ and $Y_{i}$ are negatively correlated. This follows from the definition of covariance:

$$
\begin{aligned}
& \operatorname{cov}\left[X_{i}\left(2 \mu-X_{i}\right)\right]=\mathbb{E}\left[X_{i}\left(2 \mu-X_{i}\right)\right] \\
& -\mathbb{E}\left[X_{i}\right] \mathbb{E}\left[2 \mu-X_{i}\right] \\
& =\mathbb{E}\left[X_{i}\right]\left(\mathbb{E}\left[2 \mu-X_{i}\right]-\mathbb{E}\left[2 \mu-X_{i}\right]\right) \\
& =0 .
\end{aligned}
$$

\section{Knock-Out Barrier Option Pricing using the MCS Methods}

Consider the asset price dynamics described by the stochastic differential equation below:

$$
d S(t)=S(t)(r d t+\sigma d B(t))
$$

where, $B(t)$ is the Brownian motion defined in the riskneutral probability measure and $r$ the risk-free interest rate. Using Ito's lemma to solve the above 3.1, we have: 


$$
S(t)=S(0) \exp \left(\left(r-\frac{\sigma^{2}}{2}\right) t+\sigma B(t)\right)
$$

From Equation 3.2, we have that $B(t) \sim$ $\sqrt{t} \epsilon \Rightarrow B(\Delta t) \sim \sqrt{\Delta t} \epsilon$ and $\square$ follows a standard normal distribution with the parameters $\square \sim N(0,1)$. Next, we consider the discredited underlying asset price process defined by:

$$
S^{+}(t+\Delta t)=S(t) \exp \left(\left(r-\frac{\sigma^{2}}{2}\right) \Delta t+\sigma(\sqrt{\Delta t}) \epsilon\right)
$$

The payoffs are introduced next which would be discounted at a risk-free interest rate and this depends on the type of knock-out call options being considered. For the zero rebate DO barrier call options, the discounted payoff is:

$$
v^{+}(t+\Delta t)=e^{-r \Delta t} \max \left\{S^{+}(t+\Delta t)-K, 0\right\}
$$

Equation 3.4 above occurs when the barrier is not triggered (that is, when $\max \left\{S^{+}(t+\Delta t)\right\}>B$ ) but pays zero when it knocks out (that is, when $\max \left\{S^{+}(t+\Delta t)\right\} \leq B$ ). Finally, the option value is constructed by dividing the sum of the discounted payoff by the number of simulations. Thus, the value of the Monte-Carlo simulated DO call option is given by:

$$
V_{M}=\frac{1}{M} \sum_{i=1}^{M} v^{+}(t+\Delta t)
$$

NB: Denote the Monte-Carlo simulated UO call value to be $V_{M}^{*}$, which follows the same process as discussed above. The only difference is that equation (3.4) occurs when $\max \left\{S^{+}(t+\Delta t)\right\}<B$ and zero when $\max \left\{S^{+}(t+\Delta t)\right\} \geq B$.

\section{Knock-Out Barrier Option Pricing using the AMCS Methods}

For the AMCS concept of option pricing, another set of asset price process is equally simulated so that the two processes would be negatively correlated. An average would be taken and this serves as the final simulated asset price. A more accurate result is obtained from the use of these antithetic pairs since the true option value is bounded by the positive and the negative pairs. Also, the generation of the pairs is computationally cheaper. Thus, consider two discretized underlying asset processes defined by:

$$
S^{+}(t+\Delta t)=S(t) \exp \left(\left(r-\frac{\sigma^{2}}{2}\right) \Delta t+\sigma(\sqrt{\Delta t}) \epsilon\right)
$$

And:

$$
S^{-}(t+\Delta t)-S(t) \exp \left(\left(r-\frac{\sigma^{2}}{2}\right) \Delta t-\sigma(\sqrt{\Delta t}) \epsilon\right)
$$

Next, using the risk-neutrality condition of asset pricing, these payoffs are discounted and this depends on the type of options being considered. For the zero rebate DO barrier call options, the discounted payoffs are:

$v^{+}(t+\Delta t)=e^{-r \Delta t} \max \left\{S^{+}(t+\Delta t)-K, 0\right\}$

And:

$v^{-}(t+\Delta t)=e^{-r \Delta t} \max \left\{S^{-}(t+\Delta t)-K, 0\right\}$.

Equations 3.7 and 3.8 above occur when the barrier is not triggered (that is, when $\max \left\{S^{+}(t+\Delta t)\right\}>B$ ) but the option pays zero when it knocks out (that is, when $\left.\max \left\{S^{+}(t+\Delta t)\right\} \leq B\right)$. The UO call option on the other hand pays the above when $\max \left\{S^{+}(t+\Delta t)\right\}<B$ and zero when $\max \left\{S^{+}(t+\Delta t)\right\} \geq B$. Denote its value by $V_{A}^{*}$. Finally, the mean estimator which is the required option value is constructed by taking the average of the discounted payoffs. Thus, the value of the AMCS DO call option is given by:

$$
V_{A}=\frac{1}{M} \sum_{i=1}^{M} \frac{1}{2}\left(v^{+}(t+\Delta t)+v^{-}(t+\Delta t)\right) .
$$

\section{Quasi Monte-Carlo Simulation}

Suppose we aim at calculating:

$$
\mathbb{E}\left[f\left(u_{1}, \cdots, u_{d}\right)=\int_{[0,1]^{d}} f(m) d m,\right]
$$

where, $u_{i}^{s}$ are sequences of independent uniformly distributed random variables. The QMCS entails using the approximation:

$$
\int_{[0,1]^{d}} f(m) d m \approx \frac{1}{n} \sum_{i=1}^{n} f\left(x_{i}\right)
$$

with the points $x_{i}^{\prime s}$ obtained deterministically in the $d$ dimensional closed unit hypercube $[0,1]^{d}$. The QMCS are based on the generation of low-discrepancy sequences such as Halton sequence, Faure sequence, Sobol sequence and this is in contrast to the pseudorandom numbers generated by the MCS. The error bound for QMCS is of the form $O\left(N^{-1}(\log N)^{d-1}\right)$ and it is in contrast to the standard MCS which is of the 
form $O\left(N^{\frac{-1}{2}}\right)$. The nodes $N$ are chosen to ensure that there exists low degree of regularity in the evaluation of the integral and the nodes are obtained from either of the sequences listed above (McLeish, 2000). Thus according to Glasserman (2004), the error bound satisfies the Koksma-Hlawka inequality which is written as:

$$
\varepsilon=\left|\int_{[0,1]^{d}} f(m) d m-\frac{1}{n} \sum_{i=1}^{n} f\left(x_{i}\right)\right| \leq v(f) D_{n}(A, p),
$$

where, $v(f)$ is the Hardy-Krause variation of function of $f$ and $D_{n}(A, p)$ is defined below.

\section{Definition 3.4}

Let $A$ be non-empty Lebesgue measurable subsets of $[0,1]^{d}$. The discrepancy of the point sets $p=\left\{x_{1}, \cdots, x_{n}\right\}$ relative to $A$ is defined as:

$$
D_{n}(A, p)=\sup \left\{\frac{\#\left\{x_{i} \in A\right\}}{n}-\lambda_{d}(A)\right\}
$$

where, $\#\left\{x_{i} \in A\right\}$ is the number of points in $A$ and $\lambda_{d}(A)$ is the Lebesgue measure of $A$. Extreme discrepancy and star discrepancy are obtained if $A$ takes the form:

$$
\prod_{i=1}^{d}\left[a_{i}, b_{i}\right) \text { and } \prod_{i=1}^{d}\left[0, a_{i}\right) \text { respectively, for } 0 \leq a_{i}<b_{i} \leq 1 \text {. }
$$

Discrepancy measures the uniformity deviation of specific sequence of points in $[0,1]^{d}$ and deterministic sequences tend to fill up the given space uniformly. The choice of low discrepancy sequences helps in speeding up the rate of convergence from $O(1 \sqrt{n})$ to $O(1 / n)$ and thus, the analysis of the discrepancy can help in measuring the error of the approximation method (See Niederreiter (1992) for further detailed description on analysis of low discrepancy sequences.). Further analysis of the error bound shows that small errors arise if $x_{i}^{\prime s}$ with small star or extreme discrepancies are used and this gives the low discrepancy sequences. For the purpose of this research, we consider the Sobol sequence. The Sobol sequence forms discrete uniform patterns in a specific hypercube using base two form. The construction of Sobol sequence can be found in (Jäckel, 2002) and in the generation of our results, we used the computer program (Ace option pricier (Beta) (http://www.acenumerics.com/optionpricer.html)). For the set of initial direction, this program used the methodology found in (Joe and Kuo, 2008) (Also see http://web.maths.unsw.edu.au/ fkuo/sobol/).

The knock-out barrier option pricing using the QMCS method follows the same procedure as the standard MCS. The only difference being that the QMCS uses deterministic sequences in the interval $[0,1]$ instead of the pseudo-random numbers resulting to the presence confidence intervals. Furthermore, either the Box-Muller method or the inverse transform method can be used to transform the low discrepancy sequences to normally distributed sequences. Thus, these sequences replace the normal pseudo-random numbers $\square$ as found in Equation 3.3 so as to ensure faster rate of convergence using error bounds which are already known.

\section{Computational Results and Analysis}

This section considers some of the findings obtained during the implementation of the above methodologies. For the knock-out barrier options, we consider the nondividend DO and UO call options with a zero-rebate when the option is knocked out. First, we review the results presented in Wang and Wang (2011) where they considered different Monte-Carlo methods in the valuation of barrier options. The parameters $K=50, r=$ $0.1, \sigma=0.2, T=1$ were used to value the DO put options. We compare their results on AMCS and the normal MCS using $M=10000$, with our QMCS value using $2^{13}=8192$ paths. The following table is obtained.

Table 1 compares the MCS, AMCS and the QMCS in the valuation of the DO put option with zero dividend. We chose the paths for the QMCS which are relatively lower than the number of simulations of other Monte-Carlo methods and yet, we observe that the QMCS performed outstandingly better in comparison with others, followed by the AMCS. The efficiency of the AMCS can be seen in the reduction of the variances of the MCS and this actually improved the results of the simulation. The standard errors are depicted in the brackets and we observed that the standard error for the MCS are much higher when compared to the AMCS and the QMCS. Infact, we observed that the QMCS need quite a few number of steps (as small as $2^{10}=1024$ steps) for convergence to ensue. For the computation time, the MCS took an average of $0.016 \mathrm{sec}$ for a simulation number of 10000 , to output its results. The AMCS took an average of $0.031 \mathrm{sec}$, which is about twice that of the MCS. This was because the AMCS had to reduce the variances first before the actual result was obtained. Finally, the QMCS used an average of $0.015 \mathrm{sec}$ in conjunction with $2^{10}=1024$ steps, to output its results. Thus, the QMCS most a times offer higher accuracy with lesser computation time and this outperforms the results of the MCS.

\section{Results on Zero-Rebate DO Barrier call Options with at-the-Money (ATM) Features}

We consider the values obtained using MCS, AMCS and QMCS for the above-named option which pays no dividend. Here, we consider the parameters: $S=K=150$, $B=125, r=0.05, \sigma=0.25, T=0.5$. The extended Black-Scholes formula in Equation 2.9 is used to obtain 
Nneka Umeorah and Phillip Mashele / Journal of Mathematics and Statistics 2018, Volume 14: 94.106 DOI: $10.3844 /$ jmssp.2018.94.106

the exact price and the value is 12.1861 . Figure 1 and 2 display the results obtained.

Table 1: Simulated values for DO barrier put option

\begin{tabular}{llllll}
\hline S & B & Accurate & MCS & AMCS & QMCS \\
\hline 50 & 40 & 0.6264 & $0.7101(0.0171)$ & $0.6953(0.0119)$ & $0.6264(0.0000)$ \\
55 & 40 & 0.4192 & $0.4607(0.0140)$ & $0.4527(0.0099)$ & $0.4192(0.0000)$ \\
45 & 40 & 0.6054 & $0.7306(0.0174)$ & $0.7266(0.0123)$ & $0.6054(0.0000)$ \\
50 & 45 & 0.0629 & $0.0899(0.0046)$ & $0.0858(0.0032)$ & $0.0629(0.0000)$ \\
50 & 35 & 1.4404 & $1.5063(0.0288)$ & $1.4966(0.0203)$ & $1.4404(0.0000)$ \\
50 & 30 & 1.8136 & $1.8689(0.0343)$ & $1.7969(0.0237)$ & $1.8136(0.0000)$ \\
\hline
\end{tabular}

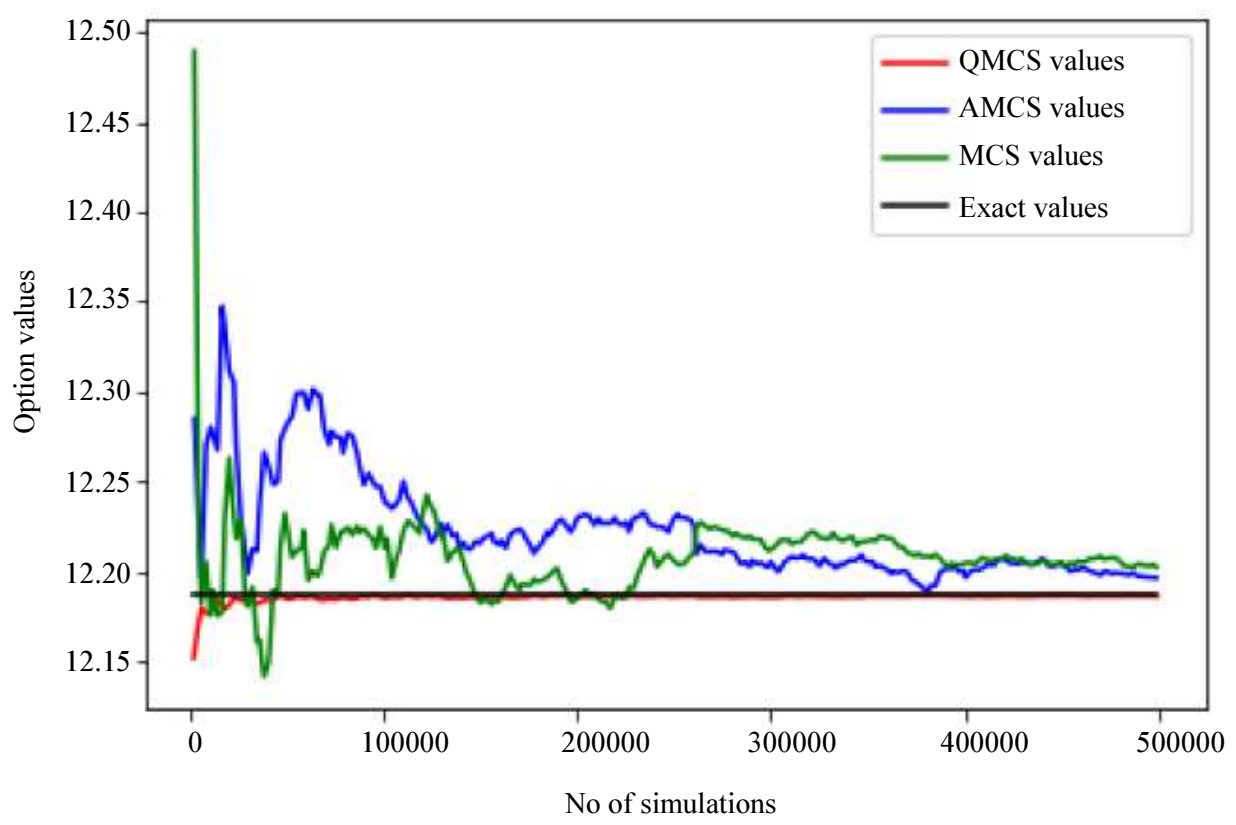

Fig. 1: Option values

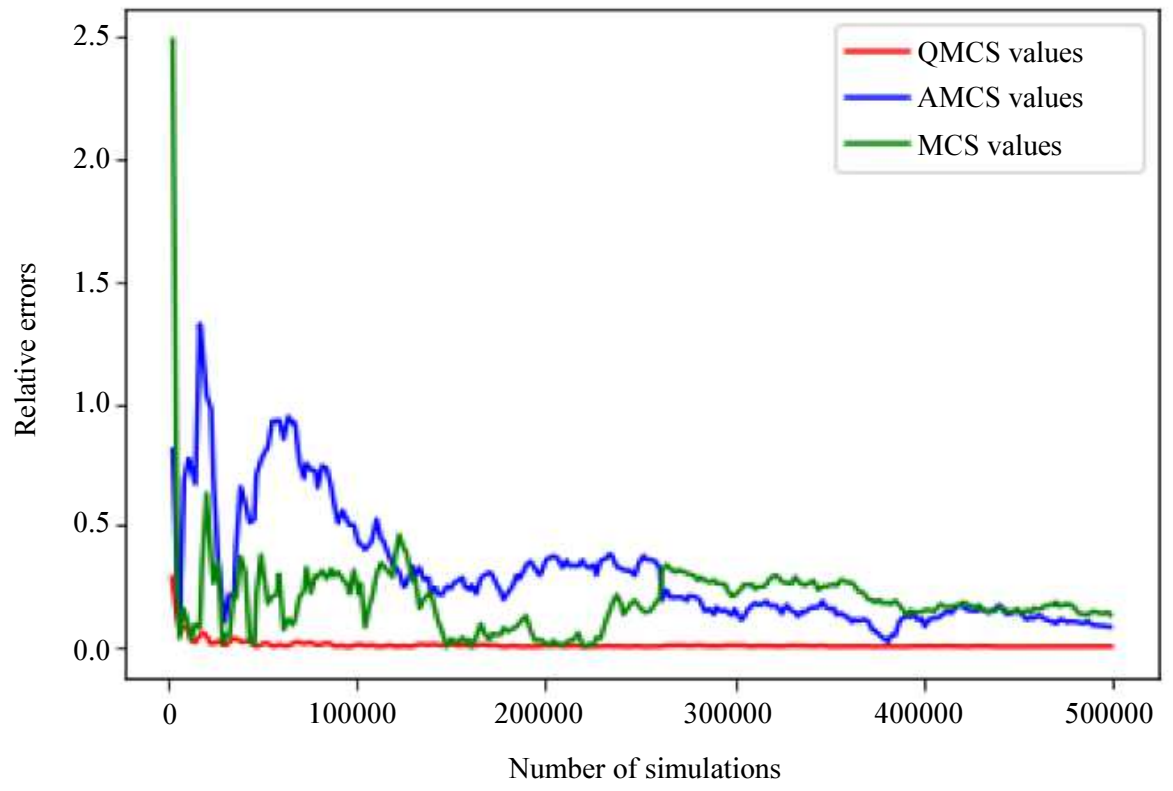

Fig. 2: Relative errors 
Figure 1 shows convergence of the values of barrier option prices when the asset price process is being simulated using various Monte-Carlo forms. The exact value remains unchanged, as it is unaffected by the number of simulations. The QMCS performed extremely well, as convergence is achieved faster and this was in connection to the use of Sobol sequences. This is followed by the performance of the AMCS, which reduced the variance of the ordinary MCS to achieve its accuracy. Figure 2 represent the plot of the relative error in percentage between the simulated values and the exact Black-Scholes price. It is calculated by:

$$
\text { Rerror }=100 \% \frac{|S I V-E B S|}{E B S},
$$

where, $E B S$ denotes the value of the exact Black-Scholes prices values and SIV is the Monte-Carlo simulated values (which could be the MCS, AMCS, or the QMCS values). We observed that the QMCS is more efficient since more consistency is achieved in the deviation of the simulated prices from the exact price.

\section{Results on the UO Call Options using MCS and AMCS Methods}

Consider the non-dividend UO call option with parameters: $S=50, K=60, B=80, r=0.05, \sigma=0.45, T$ $=0.5$. The exact value of the option using the extended Black-Scholes pricing formula is 0.8657 . We used different time points $N=100, N=1000$ and $N=10000$. The number of simulations was also considered when $M$ $=10^{1}, 10^{2}, \cdots, 10^{5}$ for each of the time points. The outputs generated are depicted graphically in the Fig. 3 and 4.
Figure 3 and 4 denote the MCS values and the AMCS values respectively, for different discretisation time points $\mathrm{N}$. The points in the graph are the centre of our discussion, as they denote the option values at each of the specified simulation number. The points are interpolated using spline curve fittings. The straight line which is unaffected by the simulation numbers denotes the option value (0.8657). In each of the figures above, we compare the estimated values obtained when the time points are $N$ $=100,1000$ and 10000 . We observed that increase in the number of simulations and increase in the time points would result to a high rate of convergence of the estimated values to the exact value, for both the MCS and the AMCS values. But the rate of convergence of the AMCS is faster when compared to the MCS since at $M=10^{5}$ and $N=10000$, the AMCS converged to the true solution.

Figures 5 and 6 denote respectively the plot of the relative errors with respect to an incremental change in the number of simulations, for both the MCS and the AMCS values. The relative errors from both simulations are reducing with an increase in the number of simulations and time points. The relative errors obtained using the AMCS are generally lesser when compared to that of MCS. The exact Black-Scholes price for the zerorebate barrier options is obtained based on continuous monitoring time. Our approach entails solving the pricing formula using the discrete time framework. This price can be achieved when the time steps $(\Delta T)$ are reduced drastically and this follows from the increment in the number of the discretisation time points $N$. Hence, the discreet points $\max _{0 \leq j \leq N} S(j)<B$ tends towards the continuous points $\max _{0 \leq t \leq T} S(t)<B$.

Table 2: Monte-Carlo simulated values for the knock-out call options

\begin{tabular}{|c|c|c|c|c|c|c|c|c|}
\hline \multirow[b]{3}{*}{ Asset (S) } & \multicolumn{8}{|c|}{ Option values } \\
\hline & \multicolumn{4}{|c|}{ DO values } & \multicolumn{4}{|c|}{ UO values } \\
\hline & Exact & MCS & AMCS & QMCS & Exact & MCS & AMCS & QMCS \\
\hline 10 & 0.0082 & $\begin{array}{l}0.0080 \\
(2.439)\end{array}$ & $\begin{array}{l}0.0081 \\
(1.220)\end{array}$ & $\begin{array}{l}0.0082 \\
(0.000)\end{array}$ & 0.0082 & $\begin{array}{l}0.0080 \\
(2.439)\end{array}$ & $\begin{array}{l}0.0081 \\
(1.220)\end{array}$ & $\begin{array}{l}0.0082 \\
(0.000)\end{array}$ \\
\hline 13 & 0.3064 & $\begin{array}{l}0.3070 \\
(0.196)\end{array}$ & $\begin{array}{l}0.3065 \\
(0.033)\end{array}$ & $\begin{array}{l}0.3064 \\
(0.000)\end{array}$ & 0.3064 & $\begin{array}{l}0.3070 \\
(0.196)\end{array}$ & $\begin{array}{l}0.3065 \\
(0.033)\end{array}$ & $\begin{array}{l}0.3064 \\
(0.000)\end{array}$ \\
\hline 16 & 1.7403 & $\begin{array}{l}1.7419 \\
(0.092)\end{array}$ & $\begin{array}{l}1.7408 \\
(0.029)\end{array}$ & $\begin{array}{l}1.7403 \\
(0.000)\end{array}$ & 1.7398 & $\begin{array}{l}1.7413 \\
(0.086)\end{array}$ & $\begin{array}{l}1.7403 \\
(0.029)\end{array}$ & $\begin{array}{l}1.7398 \\
(0.000)\end{array}$ \\
\hline 19 & 4.2567 & $\begin{array}{l}4.2582 \\
(0.035)\end{array}$ & $\begin{array}{l}4.2569 \\
(0.005)\end{array}$ & $\begin{array}{l}4.2567 \\
(0.000)\end{array}$ & 4.2325 & $\begin{array}{l}4.2349 \\
(0.057)\end{array}$ & $\begin{array}{l}4.2331 \\
(0.014)\end{array}$ & $\begin{array}{l}4.2525 \\
(0.000)\end{array}$ \\
\hline 21 & 6.1818 & $\begin{array}{l}6.1837 \\
0.031)\end{array}$ & $\begin{array}{l}6.1819 \\
(0.002)\end{array}$ & $\begin{array}{l}6.1818 \\
(0.000)\end{array}$ & 6.0303 & $\begin{array}{l}6.0337 \\
(0.056)\end{array}$ & $\begin{array}{l}6.0310 \\
(0.012)\end{array}$ & $\begin{array}{l}6.0303 \\
(0.000)\end{array}$ \\
\hline 24 & 9.1588 & $\begin{array}{l}9.1611 \\
(0.025)\end{array}$ & $\begin{array}{l}9.1589 \\
(0.001)\end{array}$ & $\begin{array}{l}9.1588 \\
(0.000)\end{array}$ & 8.0730 & $\begin{array}{l}8.0739 \\
(0.011)\end{array}$ & $\begin{array}{l}8.0723 \\
(0.009)\end{array}$ & $\begin{array}{l}8.0730 \\
(0.000)\end{array}$ \\
\hline 27 & 12.1564 & $\begin{array}{l}12.1591 \\
(0.022)\end{array}$ & $\begin{array}{l}12.1565 \\
(0.001)\end{array}$ & $\begin{array}{l}12.1563 \\
(0.001)\end{array}$ & 8.1537 & $\begin{array}{l}8.1478 \\
(0.071)\end{array}$ & $\begin{array}{l}8.1524 \\
(0.016)\end{array}$ & $\begin{array}{l}8.1537 \\
(0.000)\end{array}$ \\
\hline 30 & 15.1561 & $\begin{array}{l}15.1591 \\
(0.020)\end{array}$ & $\begin{array}{l}15.1563 \\
(0.001)\end{array}$ & $\begin{array}{l}15.1561 \\
(0.000)\end{array}$ & 5.8175 & $\begin{array}{l}5.8146 \\
(0.050)\end{array}$ & $\begin{array}{l}5.8148 \\
(0.046)\end{array}$ & $\begin{array}{l}5.8175 \\
(0.000)\end{array}$ \\
\hline 33 & 18.1561 & $\begin{array}{l}18.1594 \\
(0.018) \\
\end{array}$ & $\begin{array}{l}18.1563 \\
(0.001) \\
\end{array}$ & $\begin{array}{l}18.1561 \\
(0.000) \\
\end{array}$ & 2.2253 & $\begin{array}{l}2.2263 \\
(0.045) \\
\end{array}$ & $\begin{array}{l}2.2266 \\
(0.058) \\
\end{array}$ & $\begin{array}{l}2.2253 \\
(0.000) \\
\end{array}$ \\
\hline
\end{tabular}




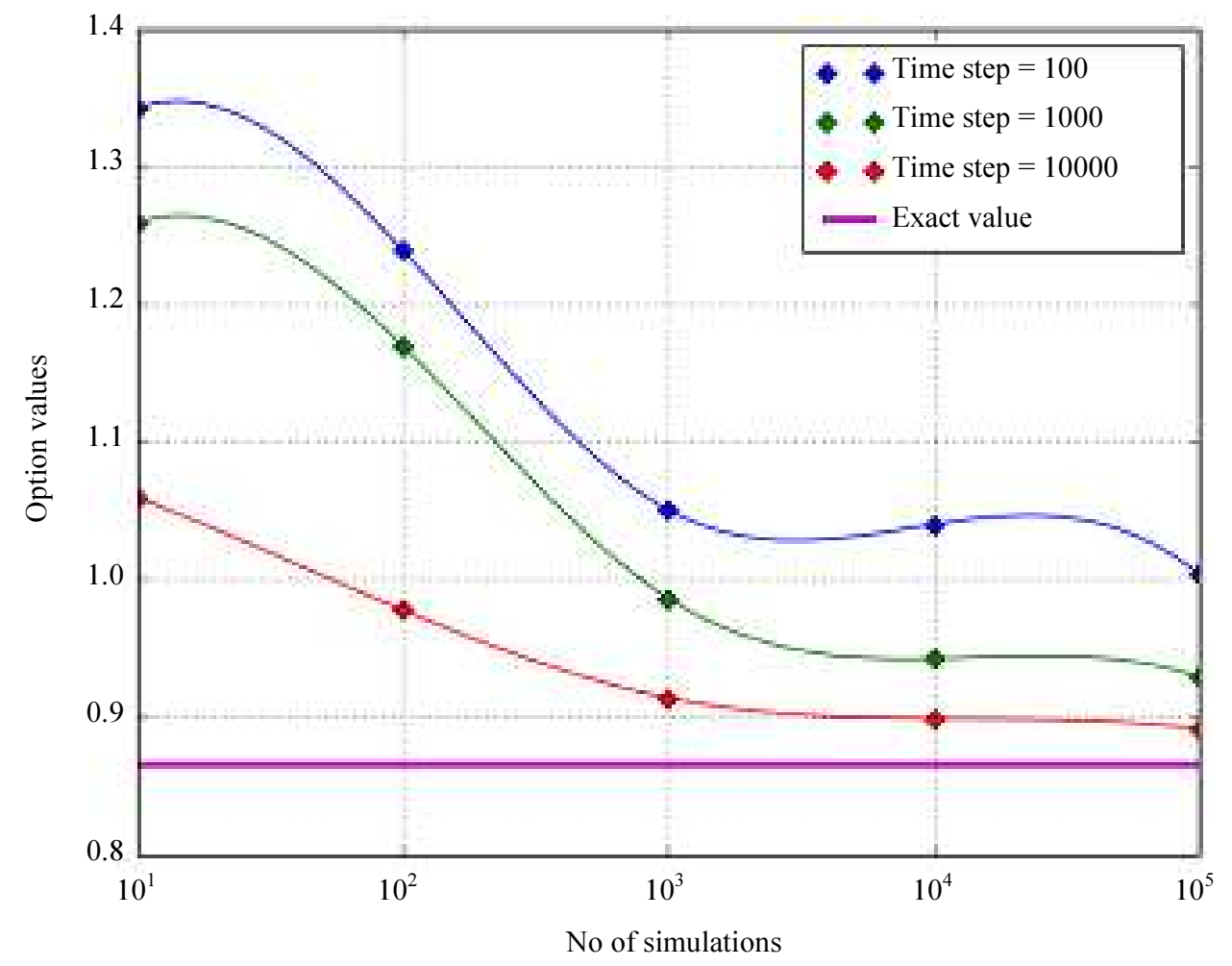

Fig. 3: MCS values with different N

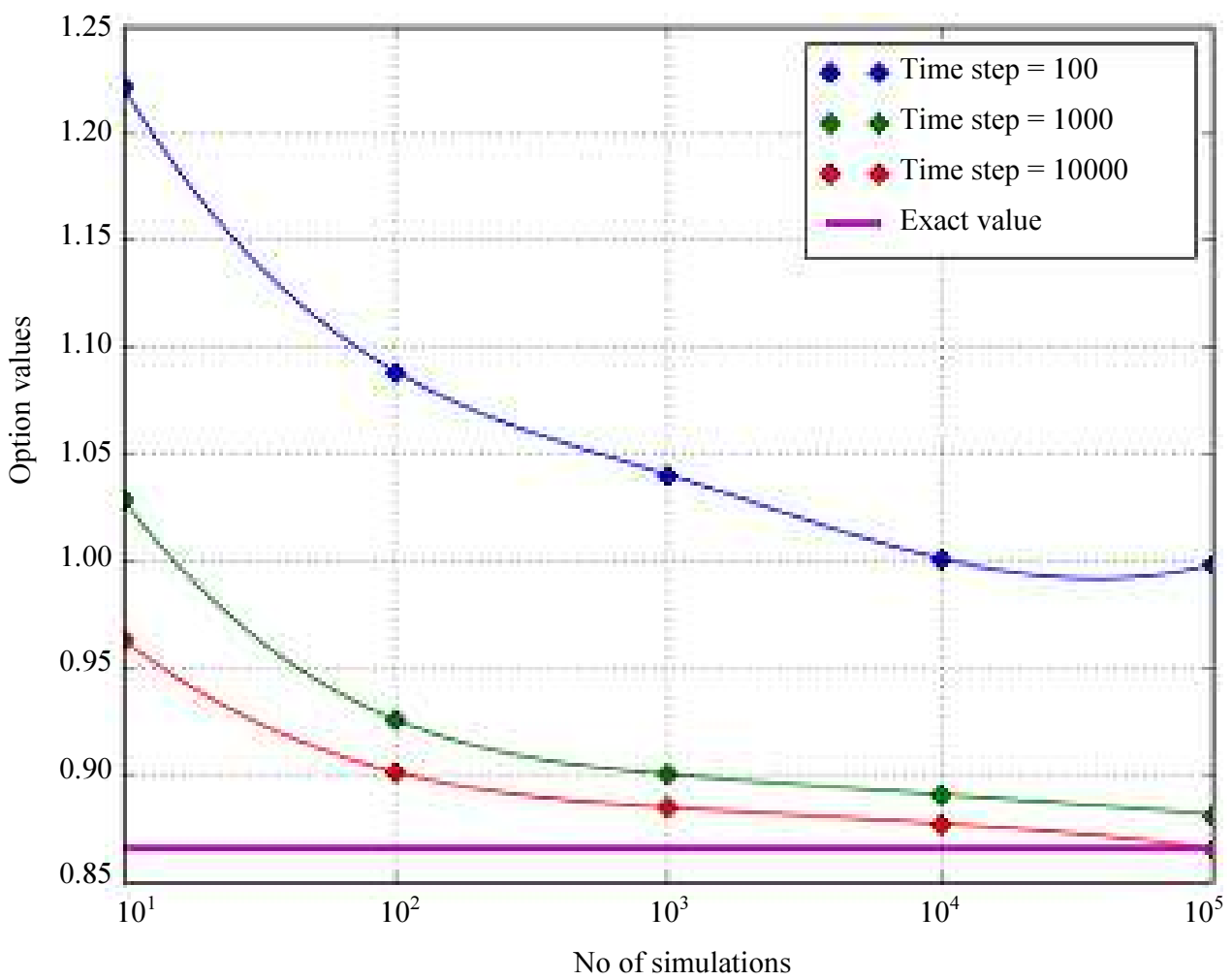

Fig. 4: AMCS values with different N 


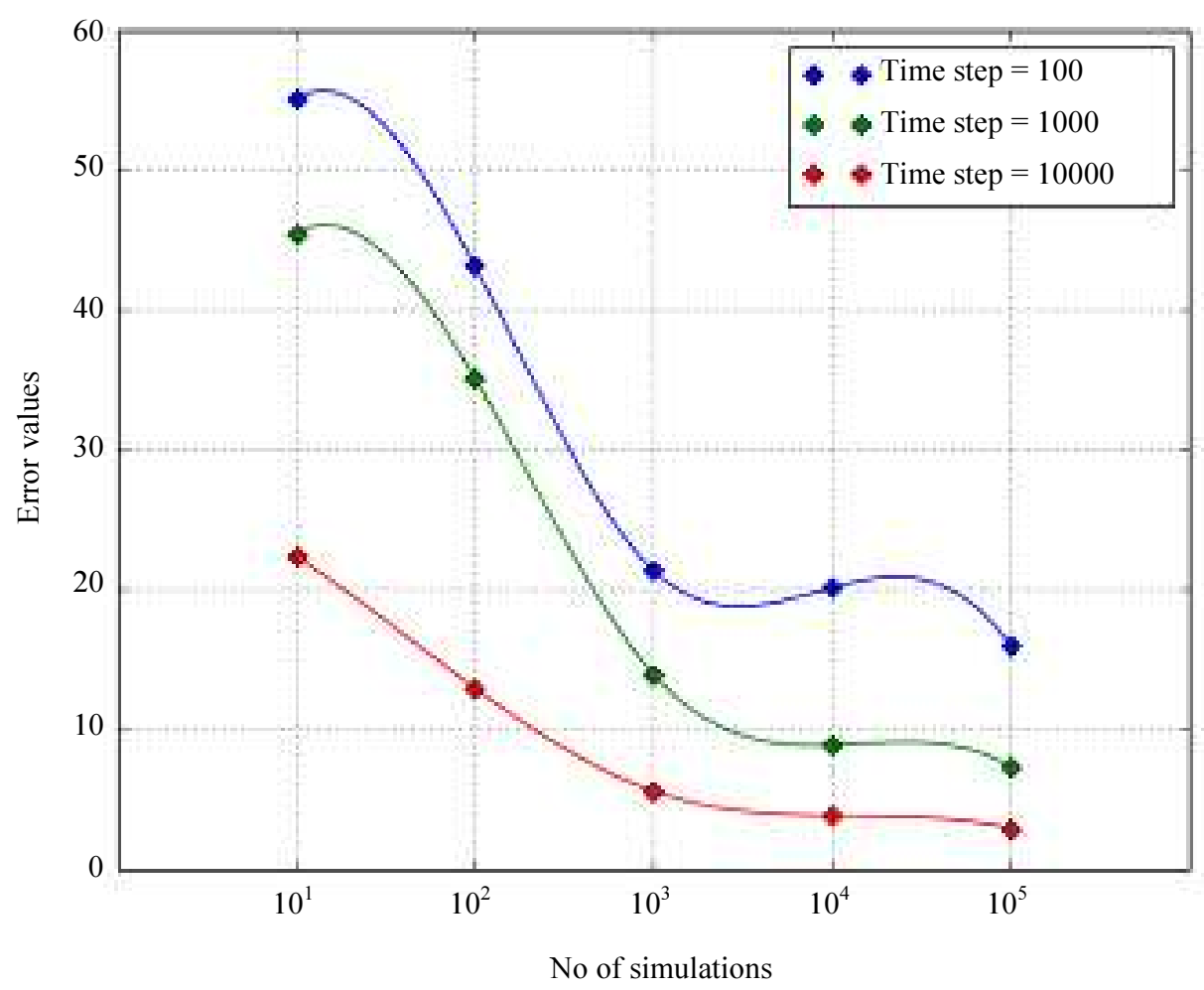

Fig. 5: Error values for MCS

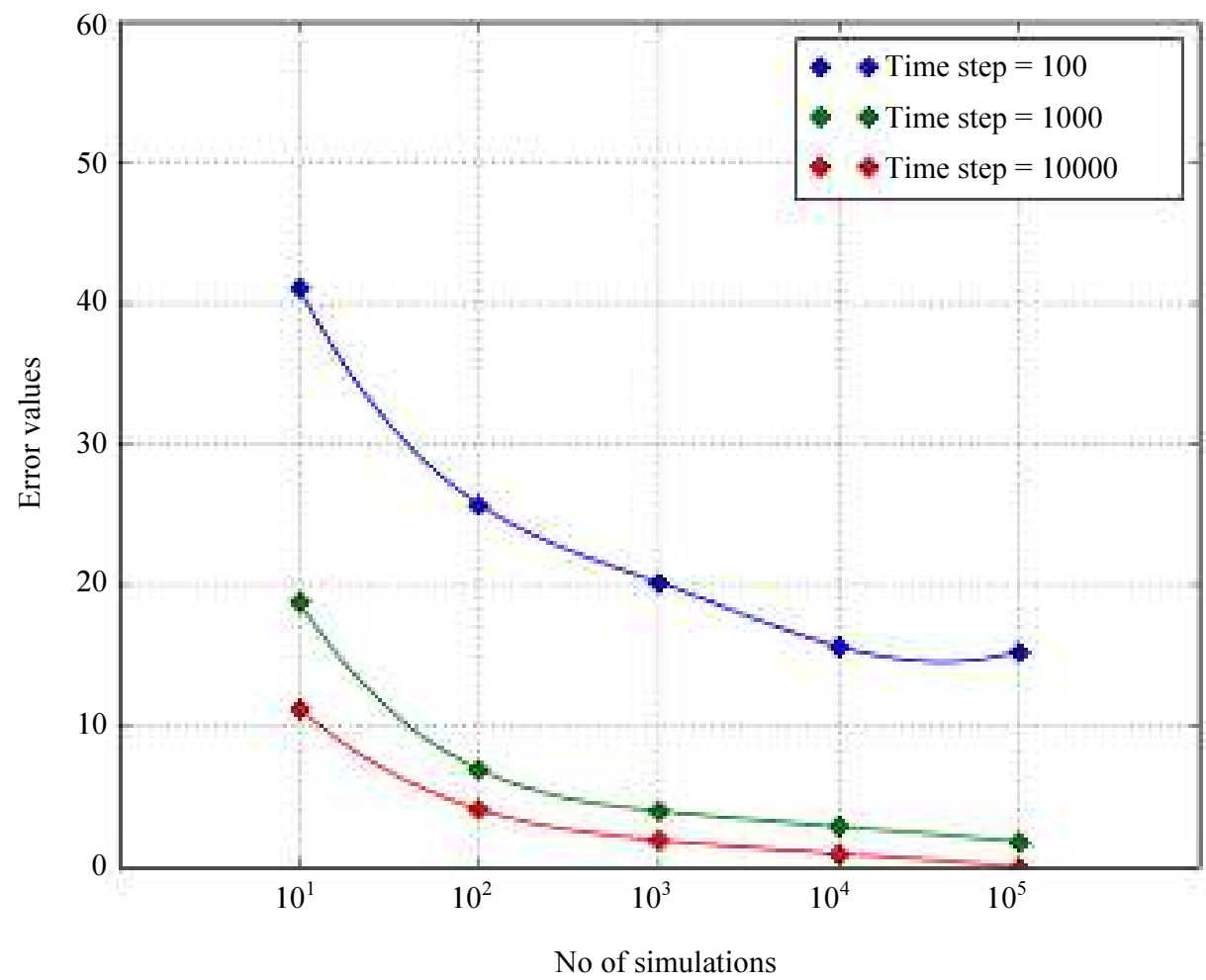

Fig. 6: Error values for AMCS 


\section{Monte-Carlo Simulation Values on Knock-Out Barrier Call Options}

Table 2 shows the comparison of the AMCS, MCS and the QMCS on the DO barrier options and the UO barrier call options. The parameters used include: $K=$ $16, r=0.1, T=0.75, \sigma=0.2$, upper barrier for $\mathrm{UO}=35$ and lower barrier for $\mathrm{DO}=5$. The following results were obtained:

From Table 2 above, we observed that the exact values for the DO options increased without bound as the underlying asset price moved away from the barrier level. Thus, the probability of the option being knocked-out was slim. Whereas, the UO call resulted in a non-linear function, as the option values increased and declined at some points. The reduction in the option values is often due to the fact that if the volatility is sufficiently large, the chances of the option being knocked out is increased. Hence, this increased probability results in the decline of the option value. The value for the UO barrier call option is always less than the difference between the barrier level and the strike price and hence it has limited upside profit potential. The values obtained using the MCS, AMCS and QMCS as depicted in the same table above show similar characteristics. The table also shows the deviation of the simulated values from the exact values. For the MCS, AMCS for both UO and DO, we consider the number of simulations to be $M=$ 600000 and $2^{19}=524288$ paths. The result shows that the AMCS is really an improved version of the MCS, with regards to their deviation from the exact value. However, when the low discrepancy method like the QMCS with sobol sequences is used to output the same option value, a highly improved version and more effective result is obtained. The relative errors are further showed in the brackets and they are expressed in percentage. It follows that the errors for the AMCS is quite smaller than that of the MCS and high rate of convergence is seen in the results obtained by the QMCS. Also, the average computation time for the QMCS to simulate $2^{19}=524288$ paths is 0.187 secs. The MCS takes an average of 0.172 secs to simulate $M=600000$ paths and AMCS takes about 0.203 secs (Recall, we used the computer program (Ace option pricier (Beta)). Hence, the QMCS outperforms the normal MCS and the AMCS.

\section{Conclusion}

A comparative study had been conducted on the valuation of non-dividend knock-out barrier options with respect to the Black-Scholes theoretical values. This research work had compared the prices of the exact Black-Scholes prices of the zero-rebate knock-out barrier options to Monte-Carlo simulated values. We have discussed results from the MCS, AMCS and the QMCS method and we observed that the ordinary MCS underpriced the option, as can be seen in the high discrepancies of the simulated values in comparison to the exact prices. It was also observed that using the AMCS resulted in the reduction of variance estimate when compared to the independent simulations obtained from the standard MCS method. This was made possible since the antithetic variables used are negatively correlated and thus, increasing one variable led to a simultaneous decrease in the other variable. Hence, the method gave a better and fairly accurate result when compared to the MCS. Furthermore, the rate of convergence is accelerated when the simulation nodes are chosen deterministically as observed by the use of low discrepancy sequences. Thus, the use of QMCS together with the Sobol sequences resulted to a more efficient method for valuing complex derivative, like the barrier options with continuous monitoring period.

Future work will extend this result to barrier options with moving barrier, as well barrier options with double barrier.

\section{Acknowledgement}

The authors wish to acknowledge the financial support from the Deutscher Akademischer Austauschdienst (DAAD) in collaboration with the African Institute for Mathematical Sciences (AIMS) and the Centre for Business Mathematics and Informatics, North-West University, Potchefstroom. We also thank the anonymous referees for their helpful comments.

\section{Author's Contributions}

Nneka Umeorah: Analysis, coding and write-up.

Phillip Mashele: Organisation and supervision.

\section{Ethics}

The authors declare that there is no conflict of interests. The corresponding author confirms that all other authors have read and approved the manuscript.

\section{References}

Alzubaidi, H., 2016. Efficient Monte Carlo algorithm using antithetic variate and Brownian bridge techniques for pricing the barrier options with rebate payments. J. Math. Stat., 12: 1-11. DOI: $10.3844 / \mathrm{jmssp} .2016 .1 .11$

Au Yeung, J., 2010. Path-dependent options pricing: A quasi Monte Carlo simulation approach with MATLAB.

Black, F. and M. Scholes, 1973. The pricing of options and corporate liabilities. J. Political Economy, 81: 637-654. DOI: $10.1086 / 260062$ 
Bolia, N. and S. Juneja, 2005. Monte Carlo methods for pricing financial options. Sadhana, 20: 347-385. DOI: $10.1007 / \mathrm{BF} 02706251$

Boyle, P., M. Broadie and P. Glasserman, 1997. Monte Carlo methods for security pricing. J. Economic Dynam. Control, 21: 1267-1321. DOI: 10.1016/S0165-1889(97)00028-6

Brandimarte, P., 2013. Numerical Methods in Finance and Economics: A MATLAB-Based Introduction. 2nd Edn., John Wiley and Sons, New York, ISBN-10: 1118625579, pp: 696.

Broadie, M., P. Glasserman and S. Kou, 1997. A continuity correction for discrete barrier options. Math. Finance, 7: 325-349. DOI: $10.1111 / 1467-9965.00035$

Buchen, P., 2012. An Introduction to Exotic Option Pricing. 1st Edn., CRC Press, ISBN-10: 142009100X, pp: 296.

Carmona, R., P. Del Moral, P. Hu and N. Oudjane, 2012. An Introduction to Particle Methods with Financial Applications. In: Numerical Methods in Finance, Carmona, R., P.D. Moral, P. Hu and N. Oudjane (Ed.), Springer Berlin Heidelberg, ISBN-10: 3642257461, pp: 3-49.

Caisch, R.E., 1998. Monte Carlo and quasi-Monte Carlo methods. Acta Numerica, 7: 1-49. DOI: $10.1017 / \mathrm{S} 0962492900002804$

Chan, N. and H. Wong, 2015. Simulation Techniques in Financial Risk Management. 2nd Edn., John Wiley and Sons, Hoboken, ISBN-10: 1118735994, pp: 232.

Cox, J.C. and M. Rubinstein, 1985. Options Markets. 1st Edn., Prentice Hall, Englewood Cliffs, ISBN-10: 0136382053, pp: 498.

Fang, K.T., 2002. Some Applications of Quasi-Monte Carlo Methods in Statistics. In: Monte Carlo and QuasiMonte Carlo Methods 2000, Fang, K.T., H. Niederreiter and F.J. Hickernell (Eds.), Springer, Berlin, Heidelberg, ISBN-10: 3642560466, pp: 10-26.

Glasserman, P., 2004. Monte Carlo Methods in Financial Engineering. 1st Edn., Springer Science and Business Media, New York, ISBN-10: 0387004513, pp: 596.

Hull, J., 2006. Options, Futures and other Derivatives. 1st Edn., Pearson Education, India, Nueva York, ISBN-10: 8131722996, pp: 822.

Jäckel, P., 2002. Monte Carlo Methods in Finance. 1st Edn., J. Wiley, Chichester, ISBN-10: 047149741X, pp: 238 .

Joe, S. and F.Y. Kuo, 2008. Constructing Sobol sequences with better two-dimensional projections. SIAM J. Scientific Comput., 30: 2635-2654.

DOI: $10.1137 / 070709359$
Kroese, D.P., T. Brereton, T. Taimre and Z.I. Botev, 2014. Why the Monte Carlo method is so important today. Wiley Interdisciplinary Rev.: Comput. Stat., 6: 386-392. DOI: 10.1002/wics. 1314

Kou, S., 2003. On pricing of discrete barrier options. Statistica Sinica, 13: 955-964.

Luenberger, D. and R. Luenberger, 1999. Pricing and hedging barrier options. Investment practice, Stanford University, EES-OR.

Merton, R., 1973. Theory of rational option pricing. Bell J. Econom. Manage. Sci. 4: 141-183. DOI: $10.2307 / 3003143$

Musiela, M. and M. Rutkowski, 2006. Martingale Methods in Financial Modelling. 2nd Edn., Springer Science and Business Media, New York, ISBN-10: 3540266534, pp: 638.

McLeish, D., 2000. Monte Carlo Simulation in Finance. 1st Edn., John Wiley and Sons.

Niederreiter, H., 1992. Random number generation and quasi-Monte Carlo methods. Society for Industrial and Applied Mathematics.

Rich, D.R., 1994. The mathematical foundations of barrier option-pricing theory. Adv. Futures Opt. Res., 7: 267-311.

Roboam, X., 2012. Systemic Design Methodologies for Electrical Energy Systems: Analysis, Synthesis and Management. 1st Edn., John Wiley and Sons, London, ISBN-10: 1118569679 , pp: 352.

Snyder, G., 1969. Alternative forms of options. Financial Anal. J., 25: 93-99. DOI: 10.2469/faj.v25.n5.93

Wang, B. and L. Wang, 2011. Pricing barrier options using Monte Carlo methods.

Wilmott, P., S. Howison and J. Dewynne, 1995. The Mathematics of Financial Derivatives: A Student Introduction. 1st Edn., Cambridge University Press, New York, ISBN-10: 0521497892, pp: 317

Zhang, P., 1998. Exotic Options: A Guide to Second Generation Options. 2nd Edn., World Scientific, Singapore, ISBN-10: 9810235216, pp: 692.

Zvan, R., K. Vetzal and P. Forsyth, 2000. PDE methods for pricing barrier options. J. Economic Dynam. Control, 24: 1563-1590. DOI: $10.1016 / \mathrm{S} 0165-1889(00) 00002-6$ 